\title{
Biosensors based on Spectral Correlation Interferometry for Biomedical Research and Diagnostics
}

\author{
P.I. Nikitin, A.V. Orlov, M.P. Nikitin, T.I. Ksenevich, B.G. Gorshkov \\ Prokhorov General Physics Institute, Russian Academy of Sciences, \\ Moscow, Russia \\ e-mail: nikitin@kapella.gpi.ru
}

\begin{abstract}
Biosensors based on the spectral correlation interferometry (SCI) with assistance of magnetic nanoparticles (MP) have been developed for medical diagnostics. The SCI has been adapted for studies of affinity constants of MP covered by antibodies and for detection of protein molecules in human serum. The 50-nm MP employed as labels yield 100-fold amplification of the SCI signals to meet the requirements for myocardial infarction diagnostics.
\end{abstract}

Keywords - biosensors; interferometry; magnetic nanoparticles; affinity constants; antibody; cardiac troponin I; autoantibodies

\section{INTRODUCTION}

Spectral correlation interferometry allows recording of changes of thickness $\Delta d$ of the interacting biomolecules on the sensor chip with picometer scale resolution averaged over the sensor channels [1]. SCI method uses standard cover glass slips as affordable single-used sensor chips (Fig.1)

A)

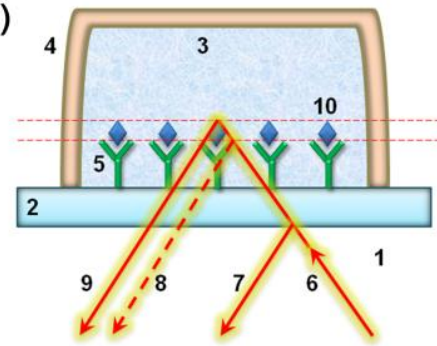

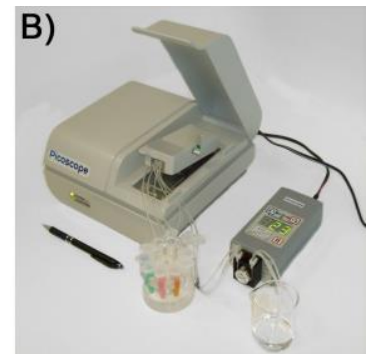

Fig. 1. The SCI principle. Changes in the thickness of a biolayer on a glass surface are recorded by a spectrum of interfering beams reflected from the sensor chip (A): 1 - air; 2 -cover slip; 3 - test solution; 4 - flow channel; 5 - receptors; 6 - incident beam of superluminescent diode; 7,9 - reflected beams; 8 - position of the reflected beam before a biochemical reaction; 10 detected biomolecules. (B): Photo of a three-channel Picoscope ${ }^{\circledR}$ biosensor.

\section{MATERIALS AND METHODS}

The MP are very attractive nanolabels for biosensors [2] Such application required knowledge of recognition properties of antibodies (AB) immobilized on MP surface. The affinity constants of $\mathrm{AB}$ coupled with MP by various methods were calculated using association model [3] adapted to the SCI. The values of the constants of association $\mathrm{k}_{\mathrm{a}}$ and dissociation $\mathrm{k}_{\mathrm{d}}$ as well as of the maximal signal $\mathrm{R}_{\max }$ were chosen to provide best fitting of the observed sensogram sections during passing the analyte at concentration $\mathrm{C}$ to the approximating function:

$$
\mathrm{R}_{\mathrm{t}}=\mathrm{Ck}_{\mathrm{a}} \mathrm{R}_{\max }\left\{1-\exp \left[-\left(\mathrm{Ck}_{\mathrm{a}}+\mathrm{k}_{\mathrm{d}}\right) \mathrm{t}\right]\right\} /\left(\mathrm{Ck}_{\mathrm{a}}+\mathrm{k}_{\mathrm{d}}\right)
$$

\section{MATERIALS AND METHODS}

The examples of sensograms for detection of protein marker of myocardial infarction - cardiac troponin I (cTnI) are shown in Fig. 2. The sensorgams demonstrate all stages of the sandwich immunoassays with using of MP as labels as well as epoxylated and biotinylated chip surfaces. Values of kinetic association constants observed at the MP stage are 2-3 orders of magnitude higher than molecular $\mathrm{AB}$ association with $\mathrm{AG}$. Such good kinetic characteristics of MP binding are explained by polyvalence of MP having several AB simultaneously.

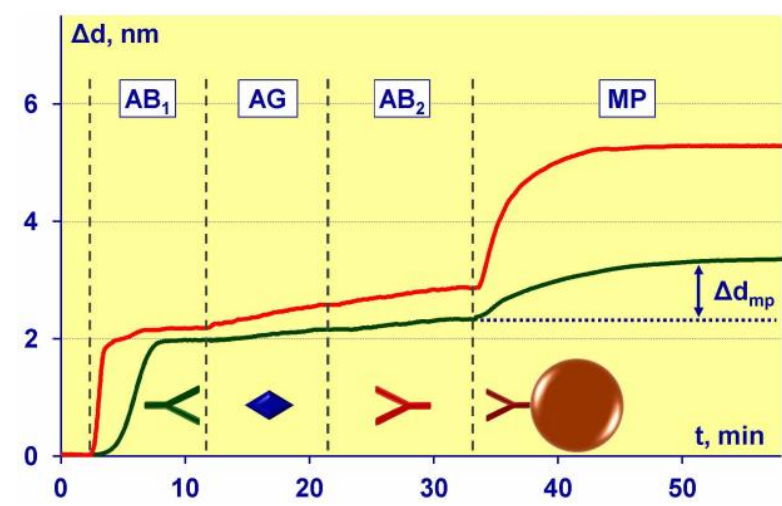

Fig. 2. Sensograms cTnI detection in label free mode (AG) and with assistance of MP on the biotinylated (bottom) and epoxylated (top) glass slips.

The 50-nm MP employed as labels yield 100-fold amplification of the SCI signals. The achieved detection limit of $\mathrm{cTnI}$ is $0.1 \mathrm{ng} / \mathrm{ml}$ meet the requirements for myocardial infarction diagnostics. The SCI biosensors functionality has been demonstrated for diagnostics of autoimmune diseases, e.g. for registration of autoantibodies against thyroglobulin and thyroperoxidase in human serum. Clinically relevant limits of autoantibodies detection are achieved at label free mode. The high sensitivity and wide dynamic range make the SCI biosensors with affordable single-used glass slips as the sensor chips the attractive instruments for medical diagnostics.

\section{REFERENCES}

[1]. P.I.Nikitin, B.G.Gorshkov, E.P.Nikitin, T.I. Ksenevich. "Picoscope, a new label-free biosensor". Sens. and Act. B, vol. 111-112, pp 500-504, 2005.

[2]. A.V.Orlov et al. Magnetic Immunoassay for Detection of Staphylococca Toxins in Complex Media. Anal. Chem. v. 85, pp. 1154-1163, 2013.

[3]. BIAevaluation 3.0 Software Handbook. Biacore AB,. P.C-2-C-33, 1997.

The research was supported by grants of Russian Foundation of Fundamental Research. 